\title{
APPLICATION OF SUPER-COMPUTER TECHNOLOGIES IN THE RESEARCH AND IMPROVEMENT OF STEPS AND ELEMENTS OF THE FLOW PATH OF THE TURBOCOMPRESSORS
}

\author{
Yuri Kozhukhov ${ }^{1, *}$, Aleksey Danilishin ${ }^{1}$, Sergey Kartashov ${ }^{1}$, Lyubov Gileva ${ }^{1}$, Aleksey Yablokov ${ }^{1}$, Vyacheslav Ivanov $^{1}$, and \\ Serafima Tatchenkova ${ }^{1}$
}

${ }^{1}$ Peter the Great St. Petersburg Polytechnic University, 195251, Saint-Petersburg, Russia

\begin{abstract}
This paper presents the results of the study of the spatial flow in the turbocompressors elements of computational fluid dynamics methods using the Ansys CFX software package on a multiprocessor computer system. Five objects of research are considered: 1) flow path of an intermediate stage of an average-flow centrifugal compressor; 2) flow path of the low-flow centrifugal compressor stage; 3) a natural gas centrifugal compressor stage; 4) vaned diffuser of the first stage of an industrial multistage centrifugal compressor; 5) adjustable inlet stator of the first stage of an industrial turbocompressors. Generally at manufacturing new centrifugal compressors, it is impossible to make a control measurement of the parameters of the working process inside the flow path elements. Computational fluid dynamics methods are widely used to overcome this difficulties. However verification and validation of CFD methods are necessary for accurate modeling of the workflow. All calculations were performed on one of the SPbPU clusters. Parameters of one cluster: AMD Opteron 280 - 4 cores. The calculations were carried out with parallel running of the processors: HP MPI Distributed Parallel and HP MPI Local Parallel for different objects.
\end{abstract}

\section{Introduction}

The essence of the study is to verify the results of modeling the working process in the elements of a centrifugal compressor, obtained using the computation fluid dynamics methods (CFD). At the Department of Compressor, Vacuum and Refrigeration Engineering (hereinafter referred to as CVRE) of SPbPU were carried out over 500 physical experiments for various elements of the flow path of a centrifugal compressor. The accumulated data bank is used for verification and validation of CFD methods. The work considers 5 different objects for verification.

\section{Methods}

The study used computational fluid dynamics methods to determine the qualitative and quantitative indicators of the working process in the elements of the flow path of a centrifugal compressor. The CFD simulation results are compared with the results of a full-scale experiment. Based on this comparison, conclusions are drawn about the further validation of methods for modeling the performance data of the flow path elements.

\section{Results}

The first object of research is the flow path of the intermediate stage of an average-flow centrifugal compressor. The objectives of the work are: a) analysis of the working process in the flow path of the intermediate stage of an average-flow centrifugal compressor using the ANSYS CFX 14.0 program with and without labyrinth seals, b) impact assessment of the frozen rotor interfaces (transfer of parameters without averaging between the rotating and stationary areas) and stage (transfer of parameters with circle averaging) for the calculation, c) analysis of the effect of a involute of the $2 \pi$ circle and verification of calculations with the results of a full-scale experiment, d) error estimation of the computational experiment.

The flow path of the compressor consists of: an axisymmetric inlet elbow, a closed-type straight blade impeller (IM), a vaneless diffuser (VLD), a return channel (RC) and labyrinth seals with intradiscal gaps. The stage was made according to the project of Yu.B. Galerkin, prof., Doctor Hub. at the Department of Compressor, Vacuum and Refrigeration Engineering of $\mathrm{SPbPU}$, under his leadership, field tests were carried out and all the necessary data for the research of the stage were provided by the authors of the article. It should be noted the wide and successful application of CFD 


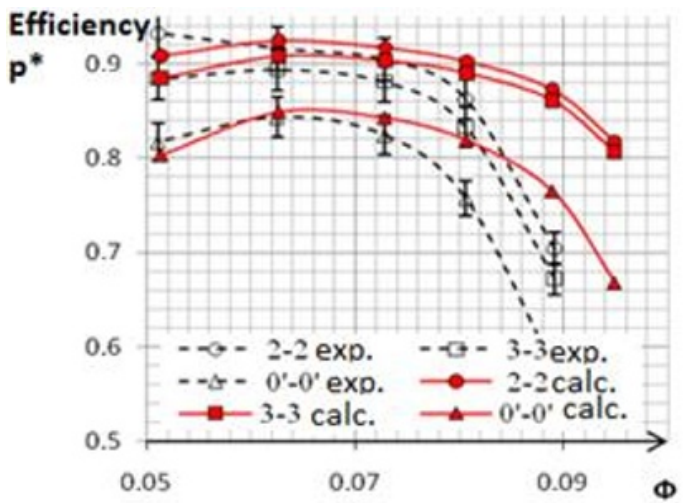

a) Verification of the result of calculating the polytropic efficiency by full parameters in three design sections for the

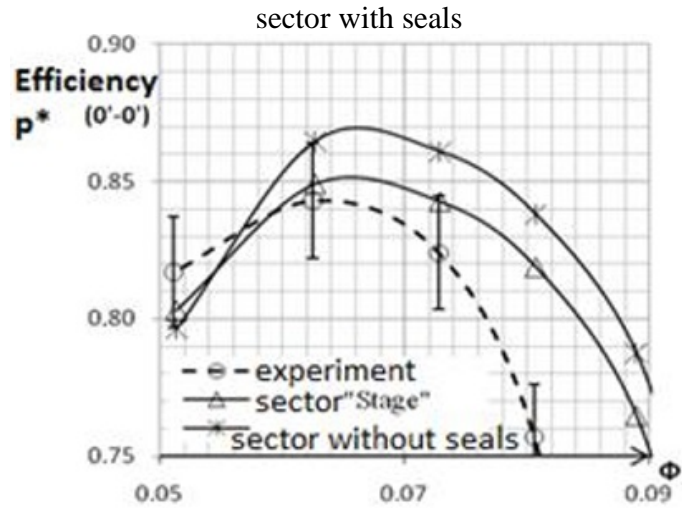

c) Impact of the presence and absence of seals on the polytropic efficiency in the calculation by full parameters for the sector

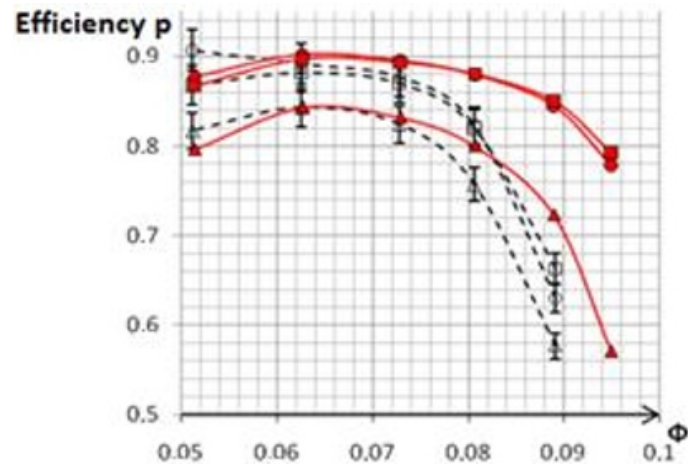

b) Verification of the result of calculating the polytropic efficiency by static parameters for the sector with seals

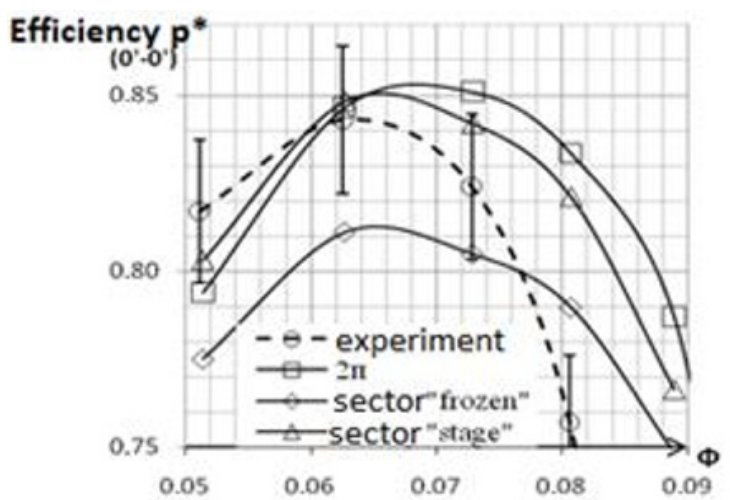

d) Comparison of the result of calculating the polytropic efficiency by full parameters for a full circle $(2 \pi)$ and sectors with «stage» and «frozen rotor» interfaces

Fig. 1. Graphs of polytropic efficiency by full and static parameters for various tasks.

methods for calculating centrifugal compressors and pneumatic systems [1-5]. The calculated grid of the flow path is: with seals - 4.47 million hexahedra, without seals - 3.51 million hexahedra, $2 \pi$ with seals - 57.6 million hexahedra. RANS is used - an approach to solving the Navier-Stokes equations, the SST turbulence model [6]. The maximum value of $\mathrm{y}+$ on $\mathrm{IM} \sim 2$.

For the preliminary calculation, from 4 to 12 nodes were used, and the calculation for 6 or more nodes did not give a significant accelerated calculation, therefore, for subsequent calculations, it was decided to use 5 cluster nodes ( 20 cores).

The calculation of 6 points of the compressor characteristic was carried out simultaneously for 300 iterations, at which the convergence of the solution was observed, which took 7 hours and 45 minutes. The calculation of the model on the full circle required significantly more resources - 24 cluster nodes, the solution of the problem took 17 hours and 45 minutes.

After the calculations, the results of the numerical experiment were processed and compared with the experimental data (Fig. 1). On the graphs of the experimental dependence, markers of the relative engineering error of the experiment are set, equal to \pm $2.5 \%$. On the charts, section 2-2 corresponds to the IM outlet at a distance of $1.05 \mathrm{D}_{2}, 3-3$ corresponds to the RC outlet, 0 '-0'corresponds to the stage outlet.
The first two graphs show good agreement of the calculation with the experimental results in the zone of the optimal operating mode. In this zone of operation, the calculation error is acceptable, but when moving to the zone of maximum flow rates, the error increases significantly. Errors arise due to the appearance of lowenergy zones - flow separations associated with the deterioration of the flow conditions around the blades in these modes, therefore, overestimation of the characteristics is observed. According to the calculation results, there is no flow separation, i.e. no energy loss.

Analysis of the influence of the initial turbulence of the flow calculated according to the inlet conditions for each mode (the kinetic energy of turbulence and the ratio of turbulent to molecular viscosity were calculated) and the roughness of the walls (in the calculation, the roughness value was set to $\mathrm{Ra}=1.6 \mu \mathrm{m}$.) The calculation showed the coincidence of the obtained characteristics.

The exclusion from the calculated region of intradiscal gaps and labyrinth seals led to the expected increase the efficiency characteristic by an average of $2.2 \%$ in section $2-2$ (Fig. 1. c), and by $1.6 \%-1.7 \%$ in the outlet section, except for one point in the region the minimum flow rate, which is associated with poor convergence at low productivity modes, since unsteady pre-surge processes appear. The calculation was carried out with the «stage» interface in the entire calculation area. 
The use of the «stage» interface in the entire computational domain (between the inlet elbow and the IM, IM and VLD, VLD and RC) led to a minimum error with the experiment, the largest error is obtained when using the «frozen rotor» interface (Fig. 1. d.). Also, a variant with the "stage» was calculated only at the inlet and outlet of the IM, the calculation of the characteristic showed that in the 0 '- 0 'section the characteristic is overestimated and decreases from higher to lower flow rates from $4 \%$ to $1.7 \%$, in other sections the characteristic is identical to the characteristic with stage parameter in all sections.

It is also of interest to calculate a full circle - a $2 \pi$ calculation that involves the use of a «frozen rotor» at the junction of the computational domains. The obtained result shows in off-design modes an underestimation by $\sim(1-2 \%)$ of the efficiency characteristics in comparison with the calculation of the sector in sections 2-2 and 3-3; in the section $0^{\prime}-0$ ', on the contrary, overestimation - this means the presence of the influence of the blade system on the flow, as well as the influence of the heterogenous nature of the distribution of parameters on the blade system and the influence of the wake behind the blades. In the design mode, there is a coincidence of characteristics, due to the best flow conditions and the absence of shock losses in the RC blade apparatus.

The second object of research is the flow path of the stage of a low-flow centrifugal compressor. The aim of the study is to obtain gas-dynamic characteristics of a low-flow stage of a centrifugal compressor with a calculated conditional mass flow rate $\Phi_{\mathrm{c}}=0.028$ for three different types of IM outlet blade edges and taking into account labyrinth seals [7] as a result of numerical modeling of a viscous gas flow and comparing the gasdynamic characteristics with the characteristics obtained as a result of a full-scale experiment. The stage studied in this work was designed, built and tested at the stand by the scientific group of Yu.B. Galerkin, prof., Doctor of Engineering Science at the CVRE Department $\mathrm{SPbPU}$. There are examples of successful application of CFD methods for low flow stages of a centrifugal compressor [8-12].

Figures 2-4 show graphs for comparing the main gasdynamic characteristics for models with labyrinth seals (LS) and without LS with characteristics obtained from the results of model tests at the CVRE Department.

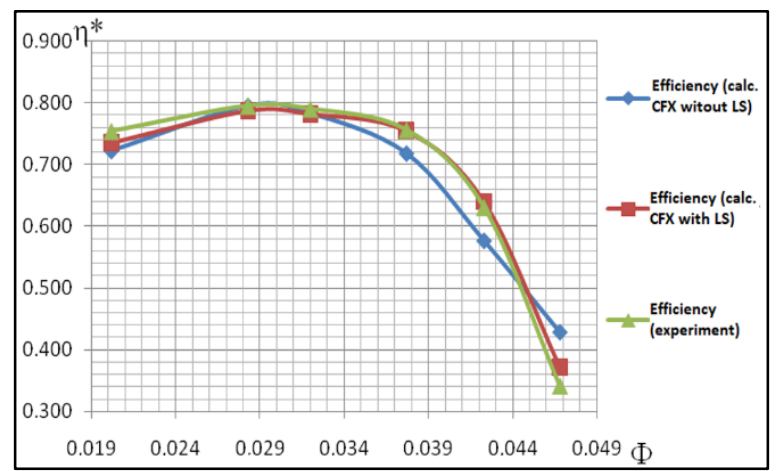

Fig. 2. The efficiency value of the CC stage in numerical and model tests (the IM blades are sharpened along the front surface to the midline).

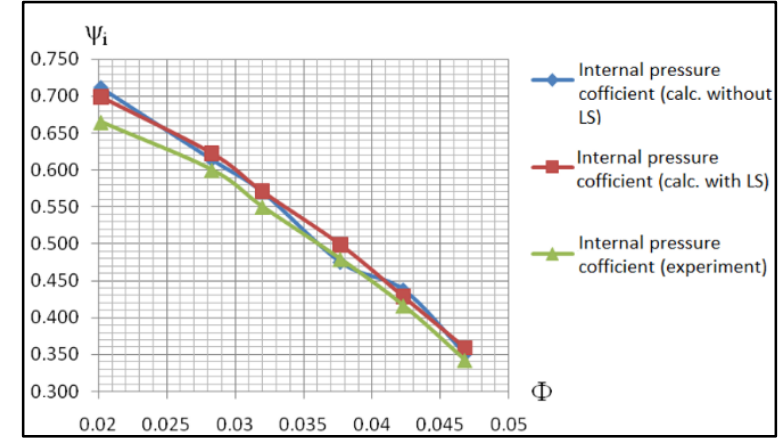

Fig. 3. The coefficient value of the internal pressure of the $\mathrm{CC}$ stage in numerical and model tests (the IM blades are sharpened along the front surface to the centerline).

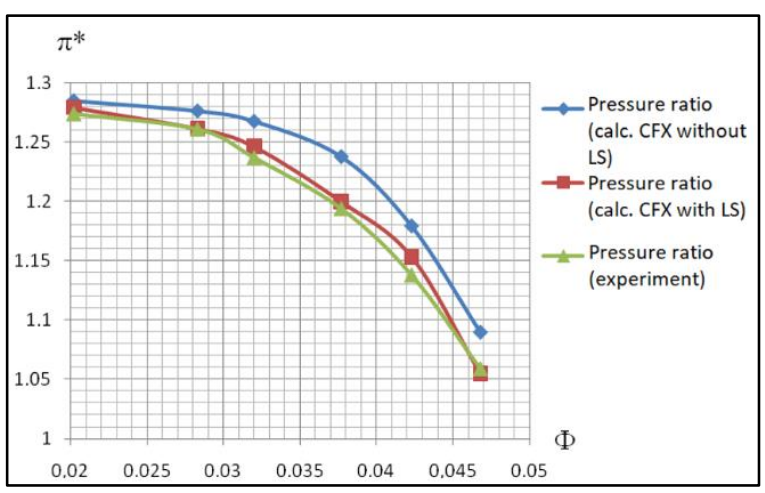

Fig. 4. The pressure ratio value of the $\mathrm{CC}$ stage in numerical and model tests (the IM blades are sharpened along the front surface to the midline).

Comparing the values of the flow characteristics and the main gas-dynamic parameters of the numerical and model experiments, it can be concluded that the model with labyrinth seals shows a better result than the simplified models. The obtained deviations values for the gas-dynamic functions are within the confidence interval and therefore the solution can be considered reliable within the goal.

The third object of research is the stage of a natural gas centrifugal compressor. The computational domain is a centrifugal compressor stage of a gas-pumping unit (see Fig. 5), consisting of IM, VLD, VD and RC. The purpose of this work is to calculate viscous threedimensional flow in Ansys CFX 14.0 to assess the characteristics of the stage. 


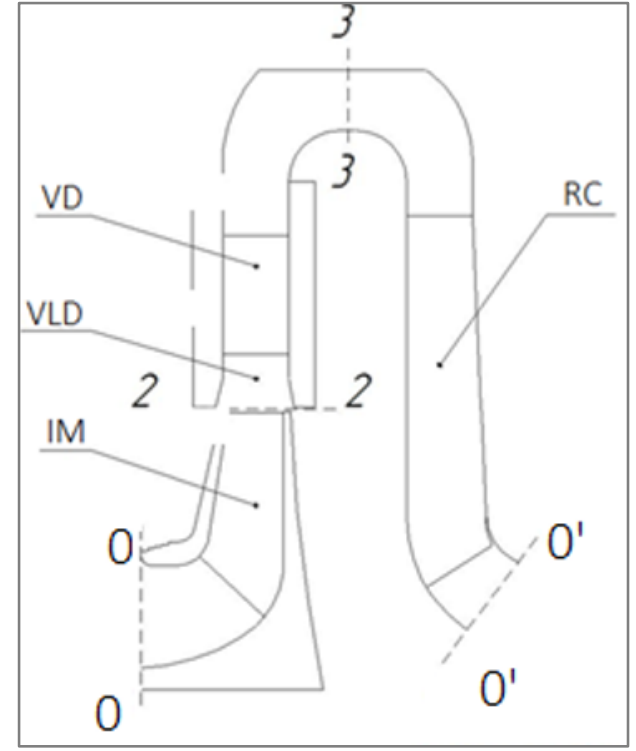

Fig. 5. Scheme of the flow path of the stage and the arrangement of control sections for processing integral parameters.

In the course of the work, structured hexahedral computational grids (4.47 million elements in total) were created, built for blade system in the Ansys Turbogrid application, and for VLD in ICEM CFD using geometric models of the flow path of a centrifugal blower provided by ZAO Nevsky Zavod.

The impeller is three-tier. The design area of the impeller consists of a sector containing 3 blades, 2 of which are cut at the inlet at different distances.

Boundary conditions for the design mode: absolute gas pressure at the inlet $-8.45 \mathrm{MPa}$, gas temperature at the inlet $-278^{\circ} \mathrm{K}$, mass flow rate at the outlet $-505 \mathrm{~kg} / \mathrm{s}$. The calculation was carried out with a stationary statement of the RANS problem (Navier-Stokes equations averaged over Reynolds). The model of shear stress transport (SST) recommended in [7] was chosen as a turbulence model.

The interfaces of the computational grids were connected according to the "Stage" criterion (averaging of parameters over the circumferential coordinate).

The calculation was carried out on two working environments:

1. Ideal methane is a perfect medium without taking into account the reality of gas, obeying the MendeleevClapeyron equation of state;

2. Natural gas - based on work [13], the equation of state "Redlich-Kwong" first published in 1949 was chosen [14], and is considered one of the most accurate two-parameter equation of state. Working fluid compressible, consisting of a mixture of the following gases: methane, ethane, propane, n-butane, n-pentane, hexane, carbon dioxide, nitrogen, water. Figures 6 and 7 show the calculated stage characteristics.

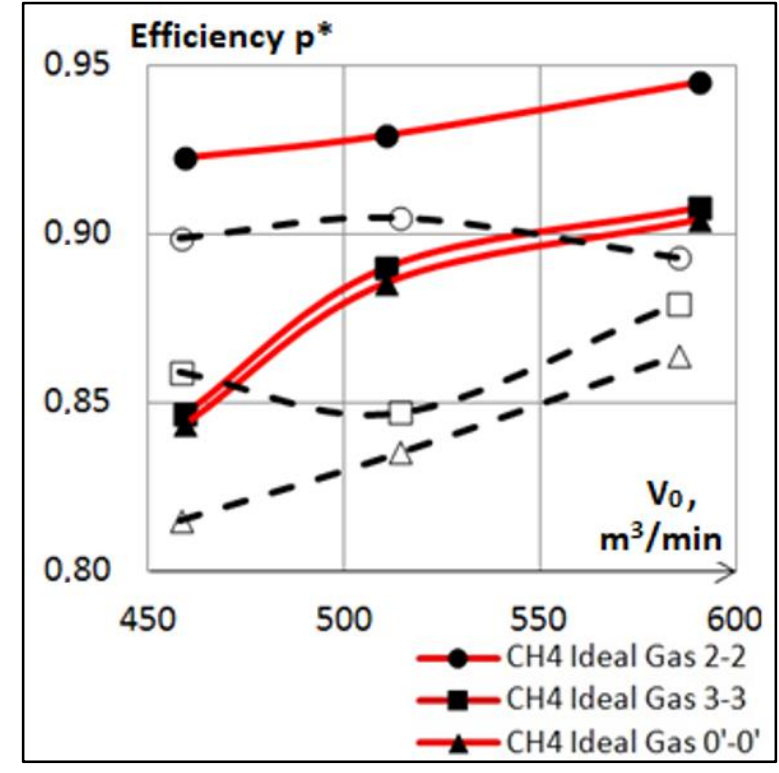

Fig. 6. Graph of polytropic efficiency values for full parameters.

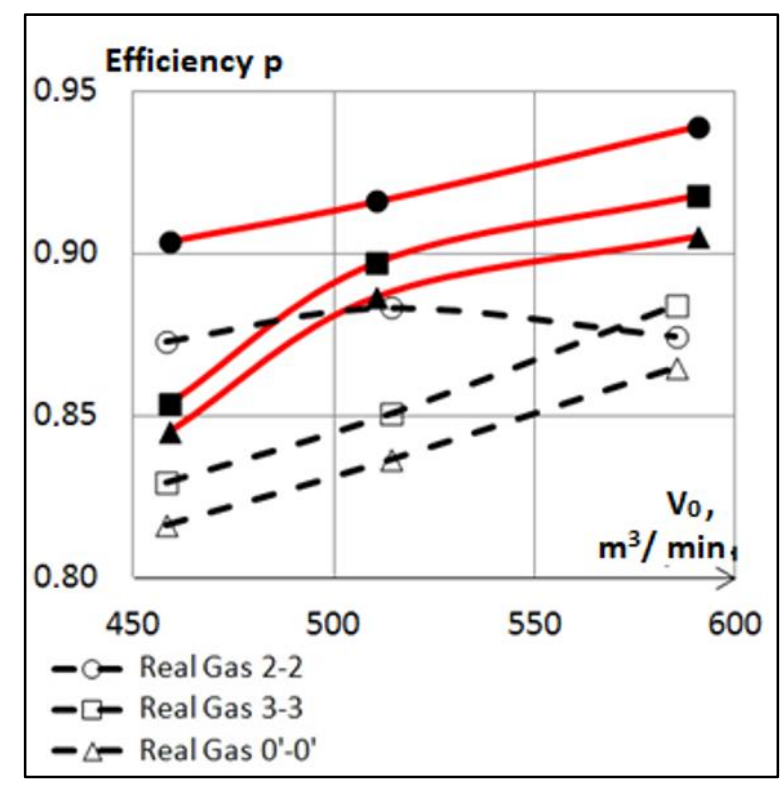

Fig. 7. Graph of polytropic efficiency values by static parameters.

After performing the calculations, the results were processed. The polytropic efficiency factors were determined for the full and static parameters.

The calculation showed the influence of reality and the multicomponent nature of the medium, which corresponds to the physics of the process; the manifestation of compressibility for methane (the main component of the working fluid) appears already at 10 atm.

The average difference in the stage efficiency is $5 \%$, in further calculations it is recommended to use a real gas, although this increases the counting time of 500 iterations at 4 nodes by 3.5 times from 8 hours 21 minutes to 30 hours 29 minutes, with an increase in the grid dimension a significant increase in counting time is excepted. 
In the future, it is planned to calculate the characteristics of the two-stage compressor and compare it with the data of full-scale tests of ZAO "Nevsky Zavod".

The fourth object of research is the vane diffuser of the first stage of an industrial multistage centrifugal compressor. The aim of this work is a numerical experiment for a vane diffuser of a centrifugal compressor stage. The studies are performed using the Ansys CFX 14.0 software package. During the study, a stationary problem was solved using the SST RANS turbulence model, according to the recommendation [15].

The flow in a vane diffuser provides a great interest to the researcher. According to [16], in a vaneless diffuser of constant width, the flow moves with an almost constant angle. If blades with an angle $\alpha_{\mathrm{bl} 1}>\alpha 3$ are installed in this vaneless space, then they will deflect the flow in the direction opposite to rotation, which will reduce the circumferential velocity component in comparison with the circumferential velocity component at the outlet from the VLD.

At the CVRE Department, research out on diffusers of centrifugal compressors using the ANSYS CFX software package is carried out, both separately and as part of a stage.

In this research paper, the question of the impact of roughness on the calculation result is considered. A vane diffuser of the first stage of a centrifugal compressor was used as an object of research. Three types of roughness were investigated: smooth walls, sand roughness 5 um and sand roughness $10 \mathrm{um}$.

The total number of elements in the entire computational domain was 1452466 elements.

As can be seen in Figure 8, a roughness $10 \mu \mathrm{m}$ gave a large separation at the exit from the blade, while smooth walls and a roughness $5 \mu \mathrm{m}$ gave a free streamline flow.

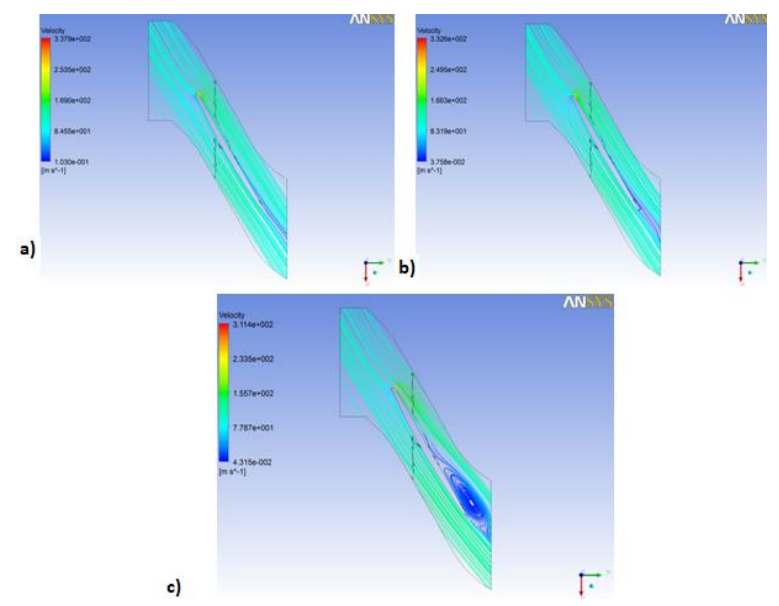

Fig. 8. Streamlines near the VD blade a) smooth walls, b) sand roughness $5 \mathrm{um}, \mathrm{c}$ ) sand roughness $10 \mathrm{um}$.

Characteristics calculations of the vane diffuser were carried out. The results are summarized in characteristics depending on the wall roughness Fig. 9.

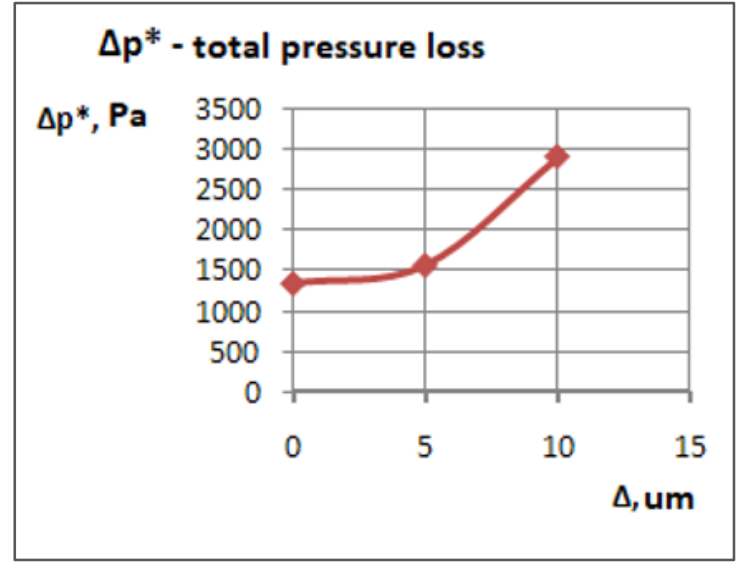

a) Dependence of total pressure loss on the wall roughness

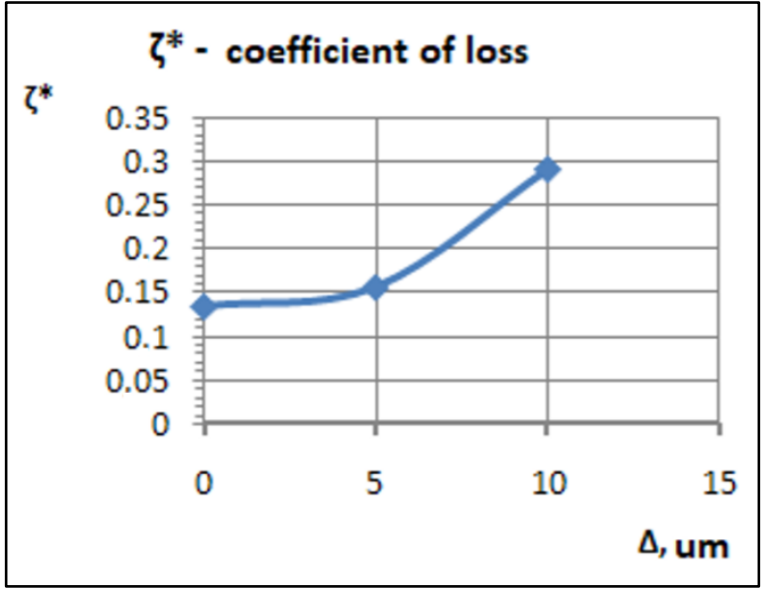

b) Dependence of the coefficient of loss on the wall roughness

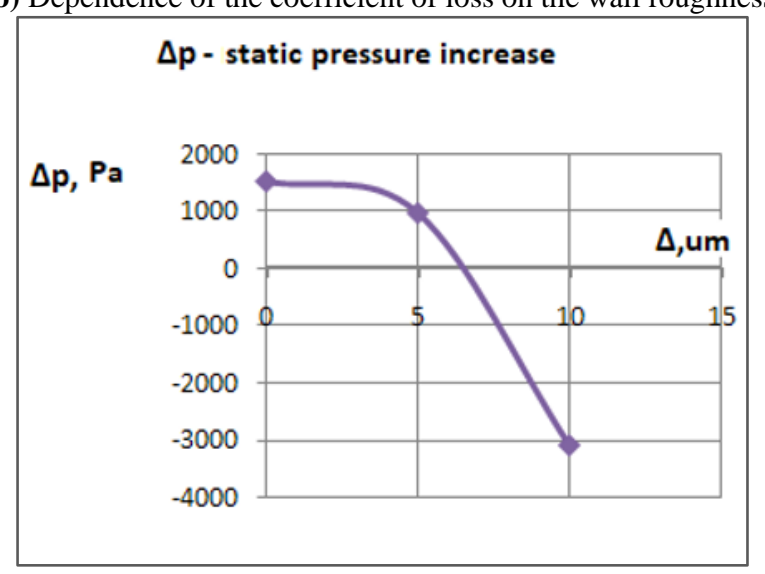

c) Dependence of static pressure increase on wall roughness

Fig. 9. a) Dependence of total pressure loss on the wall roughness; b) Dependence of the coefficient of loss on the wall roughness; c) Dependence of static pressure increase on wall roughness.

Based on fig. 8-9 and of the above it can be seen that an increase of the roughness of the walls of the vane diffuser leads to differences in the flow around the blades, an increase in the coefficient of losses and total pressure losses, as well as a reduce the static pressure increase. With a wall roughness $10 \mu \mathrm{m}$, separation is observed at the the diffuser blade outlet.

The fifth object of research is the variable inlet guide vane (VIGV) of the first stage of an industrial centrifugal compressor. VIGV is designed to create swirling air supplied to the rotor blades of the first stage 
of the compressor in order to reduce the relative velocities and obtain optimal angles of flow on the rotor blade profile. There are examples of successful application of CFD methods to the input and output devices of a centrifugal compressor $[17,18]$.

As part of a centrifugal compressor design, a study was carried out of 6 options for an variable inlet guide vane of the first stage of the compressor using computational fluid dynamics methods using the Ansys CFX 14.0 software package.

The aim of the work is to study the influence of the blades number on the coefficient of loss and the flow deviation; selection of the optimal variant of the blades number.

The study was carried out in off-design mode with a conditional mass flow rate greater than the design one and equals to $\Phi=0.099$; shock-free leakage onto the impeller (IM) blades corresponds to the VIGV installation angle $\alpha_{\mathrm{bl}}=20^{\circ}$.

When changing the number of VIGV blades, different flow patterns were obtained (Fig. 10-12).

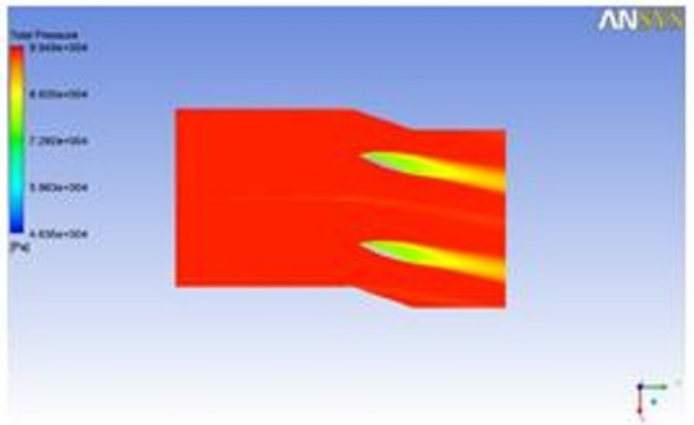

a) Distribution of total pressure.

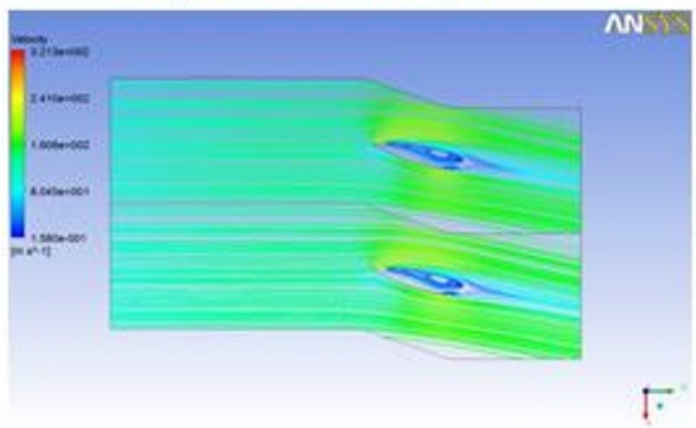

b) Streamlines and velocity values.

Fig. 10. Flow structure for VIGV with the number of blades $\mathrm{z}$ $=9$. a) distribution of total pressure; b) streamlines and velocity values.

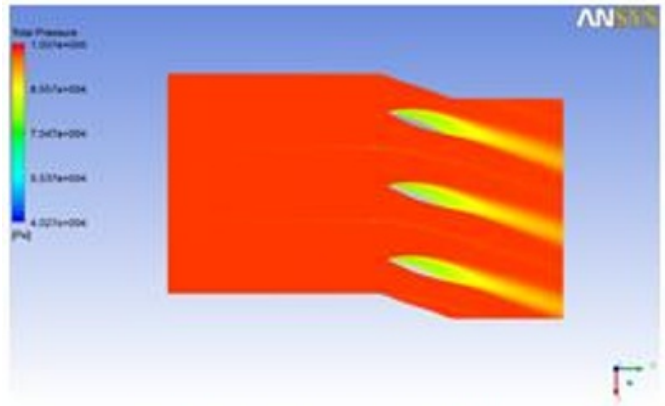

a) Distribution of total pressure.

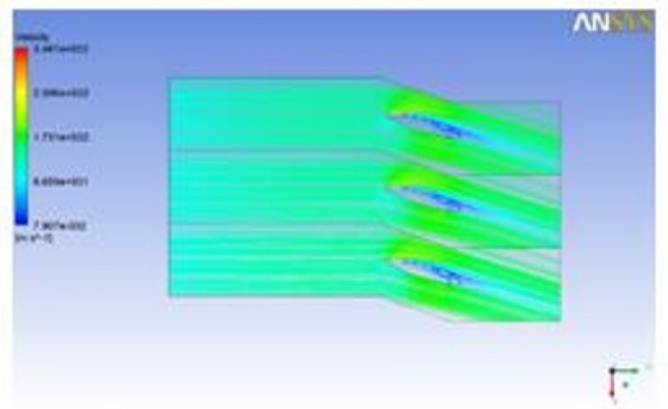

b) Streamlines and velocity values.

Fig. 11. Flow structure for VIGV with the number of blades $\mathrm{z}$ $=9$. a) distribution of total pressure; b) streamlines and velocity values.

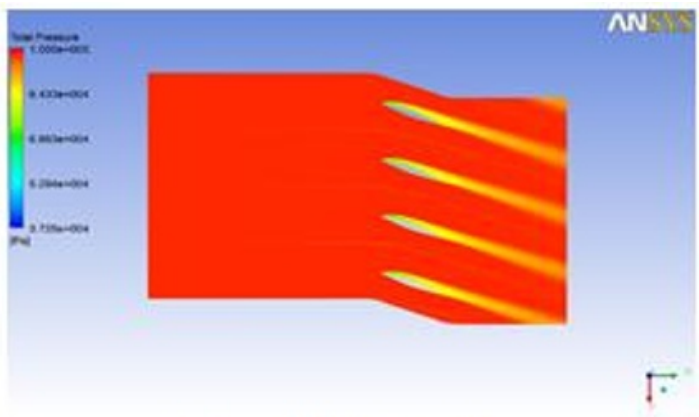

a) Distribution of total pressure.

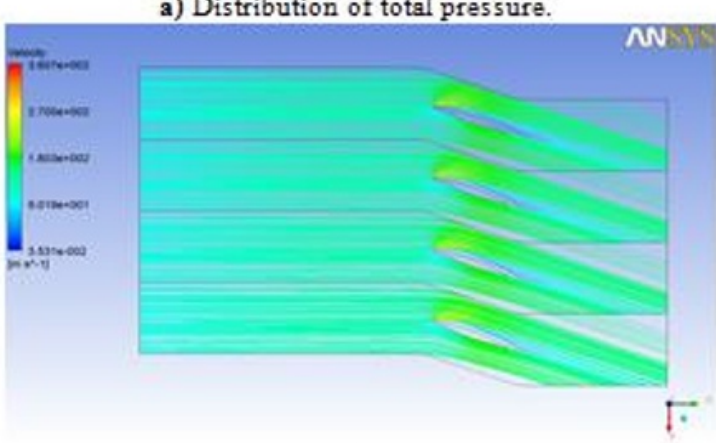

b) Streamlines and velocity values.

Fig. 12. Flow structure for VIGV with the number of blades $\mathrm{z}$ $=17$. a) distribution of total pressure; b) streamlines and velocity values.

In fig. 13-14 show the dependences of the flow deviation $\delta^{\circ}$ and the coefficient of loss for the full parameters $\varsigma^{*}$ of the VIGV depending on the number of blades z. 
The flow deviation for VIGV with the number of blades $\mathrm{z}=17: \delta^{\mathrm{o}}{ }_{17}=20-19.606=0.394$.

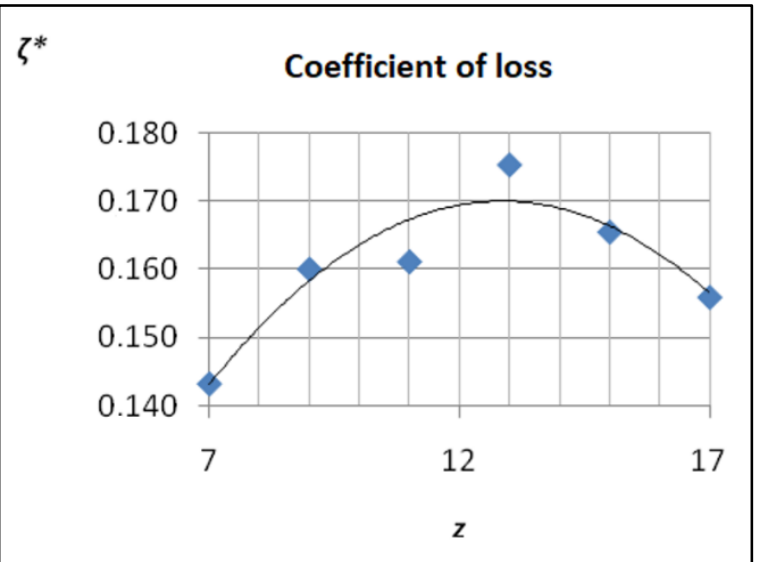

Fig. 13. The graph of the dependence of the loss coefficient $\zeta^{*}$ on the number of blades $\mathrm{z}$.

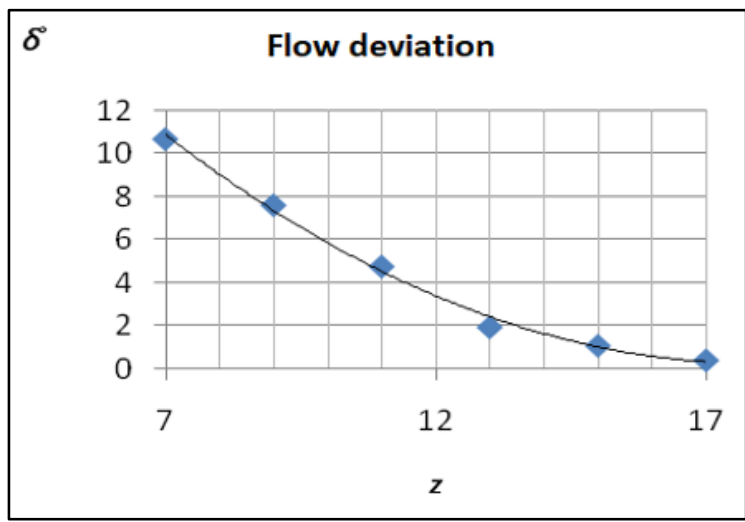

Fig. 14. The graph of the dependence of the flow deviation $\delta$ on the number of blades $\mathrm{z}$.

From the above, it can be seen that swirlings and separation are formed at the walls of the blades, which decrease to the increasing number of blades. The plotted graphs show that the lowest friction losses are for the VIGV with 7 blades, but at the same time the deviation is maximum.

The best option is VIGV with the number of blades $\mathrm{z}=17$, having a relatively low coefficient of loss and a minimum deviation. Choice could be confirmed by comparing the constraint factor $\tau$ ' for all six options and for the first stage impeller. It is necessary to ensure the flow obstruction in the VIGV is less than in the IM. This condition is necessary so that the VIGV is not a choke on the flow path to the impeller.

After analyzing the results, it can be concluded that with by increasing the number of blades in the VIGV, friction losses increase, and the deviation is reduced to a minimum.

\section{Discussions}

The use of supercomputer technologies in design and research work is becoming a sign of high quality and usefulness of research and is the most important, and in the overwhelming majority of the necessary stage of work. This allows with high accuracy and comparative speed to obtain the optimal flow path of the designed centrifugal compressor without resorting to experimental machine development.

\section{References}

1. A.A. Aksenov, A.M. Danilishin, A.M. Dubenko, Y.V. Kozhukov, Development of the virtual experimental bench on the basis of modernized research centrifugal compressor stage test unit with the 3D impeller, IOP Conference Series: Materials Science and Engineering, 232(1), 012042. (2017) DOI: $10.1088 / 1757-899 X / 232 / 1 / 012042$

2. A.A. Aksenov, A.M. Danilishin, Y.V. Kozhukhov, A.M. Simonov, Numerical simulation of gasdynamic characteristics of the semi-open 3D impellers of the two-element centrifugal compressors stages. AIP Conference Proceedings, 2007, № 030025 (2018). DOI:10.1063/1.5051886

3. P. Le Sausse, P. Fabrie, D. Arnou, F. Clunet, CFD comparison with centrifugal compressor measurements on a wide operating range, EPJ Web of Conferences, 45, 01059, (2013)

4. E. Sundström, B. Kerres, S. Sanz, M. Mihăescu, On the Assessment of Centrifugal Compressor Performance Parameters by Theoretical and Computational Models. Volume 2C: Turbomachinery (2017). doi:10.1115/gt2017-65230

5. A.M. Danilishin, S.V. Kartashov, Y.V. Kozhukhov, E.G. Kozin, The methodology for the existing complex pneumatic systems efficiency increase with the use of mathematical modelling, IOP Conference Series: Materials Science and Engineering, 232 (1), $012069 \quad$ (2017). DOI:10.1088/1757899X/232/1/012069

6. D.M. Hamburger, Numerical modeling of viscous gas flow in a centrifugal compressor stage: methodology and results, PhD thesis (2009)

7. A. Yablokov, I. Yanin, N. Sadovskyi, Y. Kozhukhov, M.H. Nguyen, Numerical characteristics of a centrifugal compressor with a low flow coefficient, E3S Web of Conferences, 140, № 6010 (2019).

DOI:10.1051/e3sconf/201914006010

8. V.M. Ivanov, Y.V. Kozhukhov, A.M. Danilishin, Calculation of the impellers head characteristics of the low-flow centrifugal compressor stages based on quasi-three-dimensional inviscid and viscous methods, AIP Conference Proceedings, 2141, 30064 (2019). DOI:10.1063/1.5122114

9. V. Ivanov, Y. Kozhukhov, M.H. Nguyen, Head Math Model for the Low-Flow Impellers of the Centrifugal Compressors, E3S Web of Conferences, 140, 6008 (2019). DOI: $10.1051 / \mathrm{e} 3$ sconf/201914006008

10. Kiryll Kabalyk, Władysław Kryłłowicz. Numerical modeling of the performance of a centrifugal compressor impeller with low inlet flow coefficient. 
ISSN 0079-3205 Transactions IFFM 131, 97-109 (2016)

11. G. Xi, Z. Wang, X. Li, S. Wang, Aerodynamic Design and Experimental Validation of Centrifugal Compressor Impellers With Small Flow Rate. Volume 7: Turbomachinery, Parts A and B (2009). doi:10.1115/gt2009-59785

12. Yongsheng Wang, Feng Lin, Chaoqun Nie, Abraham Engeda. Design and Performance Evaluation of a Very Low Flow Coefficient Centrifugal Compressor. International Journal of Rotating Machinery, 2013, Article ID 293486, 12 pages, 2013. https://doi.org/10.1155/2013/293486

13. E.G. Nikitin, Research of computational fluid dynamics methods of the spatial flow of a low-flow stage SVD-22 of a centrifugal compressor, Master thesis (2013)

14. O. Redlich, J.N.S. Kwong, On the Thermodynamics of Solutions. V. An Equation of State. Fugacities of Gaseous Solutions, (1949).

15. Garbaruk, Sagittarius, Shur, Turbulence modeling in complex flow calculations, (2012)

16. Yu.B. Galerkin, Yu.V. Kozhukhov, The theory of turbomachines. Fundamentals of the theory of turbocompressors. (2013)

17. Y.V. Kozhukhov, V.K. Yun, L.V. Reshetnikova, M.V. Prokopovich, Numerical Investigation of Different Radial Inlet Forms for Centrifugal Compressor and Influence of the Deflectors Number by Means of Computational Fluid Dynamics Methods with Computational Model Validation, IOP Conf. Ser.: Mater. Sci. Eng., 90, 012047 (2015) DOI: 10.1088/1757-899X/90/1/012047

18. A.M. Danilishin, Y.V. Kozhukhov, S.V. Kartashov, A.A. Lebedev, K.G. Malev, Y.R. Mironov, Design optimization opportunity of the end stage output plenum chamber of the centrifugal compressor for gas pumping unit, AIP Conference Proceedings, 2007, 30044 (2018). DOI: 10.1063/1.5051905. 